\title{
Prevalencia de la hipovitaminosis D en una población socioeconómica alta y su asociación con diferentes entidades nosológicas
}

\section{Prevalence of hypovitaminosis D in a high socioeconomic population and its association with different nosological entities}

\author{
Gregorio Arellano Aguilar,* Ernesto Marín y Santillán, ${ }^{\ddagger}$ \\ Dolores Vanessa Jonguitud Díaz, ${ }^{\S}$ Luis Gerardo Domínguez Carrillo"|
}

\section{Resumen}

Introducción: La vitamina D es considerada una prohormona con efectos pleiotrópicos. Su deficiencia tiene una prevalencia alta, relacionándose no sólo con raquitismo y osteomalacia, sino también con un mayor riesgo de asociación con diabetes, enfermedades cardiovasculares, oncológicas, infecciosas y autoinmunes. El objetivo es valorar los niveles de vitamina $D$ total en una población socioeconómica alta y su asociación con diagnósticos clínicos establecidos en un estudio observacional y retrospectivo de cohorte. Material y métodos: Se incluyeron pacientes entre 20 y 80 años con cuantificación de $25-\mathrm{OH}$ vitamina $\mathrm{D}$ por el método de quimioluminiscencia. Se consideró como disminución de vitamina $\mathrm{D}$ a un valor menor de $30.0 \mathrm{ng} / \mathrm{mL}$, correlacionándolo por la medida de asociación odds ratio (OR) con los diagnósticos establecidos en la solicitud de examen. Resultados: Del total de 90 pacientes, 60 pertenecieron al género femenino $(66.6 \%)$; hubo disminución de valores normales en 57 casos (63.3\%) con promedio y DE de $22.8 \pm 5.6 \mathrm{ng} / \mathrm{mL}$. Se observó una asociación con diabetes $(\mathrm{OR}=1.19)$, osteoporosis $(\mathrm{OR}=0.95)$, hipertensión arterial $(\mathrm{OR}=0.58)$, hipercolesterolemia en tratamiento $(\mathrm{OR}=0.28)$, enfermedad renal crónica $(O R=0.20)$ y otras entidades. Conclusiones: Los niveles de $25-\mathrm{OH}$ vitamina $\mathrm{D}$ se encontraron por debajo de lo normal en dos tercios de la muestra estudiada, los cuales se asociaron con varias enfermedades. En este estudio, sólo con diabetes mellitus se encuentra como factor de riesgo; respecto al resto de las entidades estudiadas, los resultados no son concluyentes.

Palabras clave: Vitamina D, déficit, enfermedades relacionadas.

\section{Abstract}

Introduction: Vitamin D is considered a prohormone with pleiotropic effects, its deficiency has high prevalence; related not only with rickets and osteomalacia, but with a diabetes increased risk, cardiovascular, oncological, infectious and autoimmune diseases. To value in an observational, retrospective cohort study, vitamin D levels in a high socioeconomic population and its association with established clinical diagnoses. Material and methods: patients between 20 and 80 years old who underwent quantification of vitamin D $25-\mathrm{OH}$ by the chemiluminescence, it deficiency was considered when the quantificacation was lower than $30.0 \mathrm{ng} / \mathrm{mL}$; correlating with diagnoses established in patients. Results: Universe of 90 patients, 60 the female gender (66.6\%); reduction of normal values in 57 cases $(63.3 \%)$ with mean and SD of de $22.8 \pm$ $5.6 \mathrm{ng} / \mathrm{mL}$; observing association with diabetes $(\mathrm{OR}=1.19)$, osteoporosis (OR $=0.95)$, arterial hypertension (OR $=0.58)$, hypercholesterolemia $(\mathrm{OR}=0.28)$, chronic kidney disease $(\mathrm{OR}=0.20)$, and others entities. Conclusions: The levels of $25-\mathrm{OH}$ Vitamin D were below normal in $2 / 3$ of the studied sample, associated with several diseases, in this study only with diabetes mellitus is found as a risk factor, the association with the rest of the entities studied is not conclusive.

Keywords: Vitamin D, deficiency, related diseases.

* Especialista en Medicina Interna. División de Medicina del Hospital Ángeles León, León, Guanajuato. México.

₹ Cirujano General. Director Médico del Hospital Ángeles León. León, Guanajuato. México.

§ Patóloga Clínica. Jefe del Laboratorio de Patología Clínica del Hospital Ángeles León. León, Guanajuato. México.

|| Especialista en Medicina de Rehabilitación. Facultad de Medicina de León, Universidad de Guanajuato. México.

Correspondencia:

Dr. Luis Gerardo Domínguez Carrillo

Correo electrónico: gregareag@gmail.com

Aceptado: 19-06-2019.

www.medigraphic.com/actamedica 


\section{INTRODUCCIÓN}

La deficiencia de vitamina $\mathrm{D}$ es considerada un problema de salud pública a nivel internacional, pues afecta a todos los grupos etarios, especialmente en países ubicados a elevadas latitudes, e incluso en los países expuestos al sol durante todo el año, en donde se esperaría que los rayos ultravioleta solares fueran suficientes para evitar su deficiencia. ${ }^{1,2}$ En el reporte multinacional de Lips y su equipo, ${ }^{3}$ en el que estudiaron a mujeres postmenopáusicas con osteoporosis provenientes de 18 países de Europa, Asia y América, se reportó una alta prevalencia de deficiencia de vitamina D en Corea del Sur (92.1\%), Japón (90.4\%), Líbano (84.9\%), Turquía (76.7\%), Reino Unido (74,5\%), Alemania (68.0\%), México (67.1\%) y España (64.7\%). Adicionalmente, se ha evidenciado que la hipovitaminosis $\mathrm{D}^{4}$ es significativamente más frecuente en los adultos mayores, cuya prevalencia oscila entre el 50 y $90 \%,{ }^{5,6}$ así como en mujeres gestantes, en donde se ha descrito que la deficiencia de vitamina $\mathrm{D}$ puede estar relacionada con peores desenlaces fetales y neonatales. ${ }^{7}$ Al respecto, en una cohorte de 7,256 pacientes que acudieron a maternidad en Rotterdam, Holanda, se encontró una prevalencia de hipovitaminosis D del $53 \%$ en las madres y del $46 \%$ en los neonatos, el cual, al compararlo con el estudio de Ochoa ${ }^{8}$ y su grupo, realizado en México, se observó que de 191 mujeres puérperas y sus neonatos, $61 \%$ de las madres presentó deficiencia de vitamina D y $26 \%$ manifestó insuficiencia, mientras que el $98 \%$ de sus neonatos a término mostró deficiencia de vitamina $\mathrm{D}$.
Con el fin de investigar en nuestro medio si existen diferencias con lo descrito en la literatura, en una población con nivel socioeconómico alto, se efectuó un estudio observacional, restrospectivo de cohorte de los datos obtenidos en la cuantificación de 25-OH vitamina $\mathrm{D}$, además de correlacionar los resultados con los diagnósticos inscritos por el médico en la solicitud de laboratorio mediante medidas de asociación.

\section{MATERIAL Y MÉTODOS}

Se solicitaron al laboratorio de la institución todas las cuantificaciones de $25-\mathrm{OH}$ vitamina $\mathrm{D}$, realizadas por el método de quimioluminiscencia, entre enero de 2017 y noviembre de 2018, en pacientes con edades comprendidas entre 20 y 80 años, independientemente del género; tomando como valores de referencia para considerarse dentro del rango normal entre 30.0 y $100.0 \mathrm{ng} / \mathrm{mL}$. La muestra se clasificó por: grupos etarios por decenios; por género; por cuantificación dentro de los valores de referencia, y aquéllos con cuantificación menor a $30 \mathrm{ng} / \mathrm{mL}$. Se calcularon medidas de asociación OR (odds ratio o razón de desventaja) con el o los diagnósticos incluidos en la solicitud de estudio.

\section{RESULTADOS}

La muestra consistió en 90 cuantificaciones de $25-\mathrm{OH}$ vitamina $\mathrm{D}$ en 90 pacientes, perteneciendo al género masculino un total de $33.3 \%(n=30)$ y al femenino un $66.6 \%$ de los casos $(n=60)$; al clasificar por grupos etarios

Tabla 1: Cuantificación de 25-OH vitamina D en 90 pacientes, con distribución por valores normales y disminuidos y su distribución por género y grupos etarios.

\begin{tabular}{|c|c|c|c|c|c|c|}
\hline Cuantificación de $25-\mathrm{OH}$ vitamina D & $\mathrm{N}-\mathrm{T}$ & $\%$ & N-M & $\%$ & $\mathrm{~N}-\mathrm{F}$ & $\%$ \\
\hline Muestras & 90 & 100.0 & 30 & 33.30 & 60 & 66.6 \\
\hline Rango normal (30 a 100 ng/mL) & 33 & 36.6 & 6 & 6.66 & 27 & 30.0 \\
\hline Valores inferiores a 30 ng/mL & 57 & 63.4 & 24 & 26.60 & 33 & 63.3 \\
\hline Grupos etarios divididos por décadas & $\mathrm{N}-\mathrm{T}$ & $\%$ & $\mathrm{~N}-\mathrm{M}$ & $\%$ & $\mathrm{~N}-\mathrm{F}$ & $\%$ \\
\hline $20-29$ & 14 & 15.5 & 0 & 0.0 & 14 & 100.0 \\
\hline $30-39$ & 8 & 8.8 & 2 & 25.0 & 6 & 75.0 \\
\hline $40-49$ & 23 & 25.5 & 5 & 21.7 & 18 & 78.3 \\
\hline $50-59$ & 15 & 16.6 & 6 & 40.0 & 9 & 60.0 \\
\hline $60-69$ & 19 & 21.1 & 12 & 63.1 & 7 & 36.9 \\
\hline $70-79$ & 11 & 12.2 & 5 & 45.4 & 6 & 54.6 \\
\hline
\end{tabular}


(decenios), se observó que la mayoría de los pacientes pertenecieron a los grupos de edad entre 41 y 70 años, alcanzando $63.3 \%$ de la muestra $(\mathrm{n}=7)$ (Tabla 1$)$.

Las cuantificaciones dentro del rango se consideraron dentro de lo normal en $41.1 \%$ de los casos ( $n=33$ ); de éstas, el $18.2 \%(\mathrm{n}=6)$ fue de sexo masculino y $81.8 \%(\mathrm{n}=$ 27) femenino; las cuantificaciones de la $25-\mathrm{OH}$ vitamina $D$ menores de $30 \mathrm{ng} / \mathrm{mL}$ correspondieron al $63.3 \%$ de la muestra $(\mathrm{n}=57)$, perteneciendo $26.6 \%$ al género masculino ( $\mathrm{n}$ $=24)$ y $36.6 \%$ al femenino $(n=33)$. Se observó promedio y DE de $22.8 \pm 5.6 \mathrm{ng} / \mathrm{mL}$ con variación de 7 a $29 \mathrm{ng} / \mathrm{mL}$. La asociación de valores bajos de $25-\mathrm{OH}$ vitamina $\mathrm{D}$ con los diagnósticos de solicitud de estudios se muestran en la Tabla 2, encontrando que las asociaciones más frecuentes fueron con diabetes mellitus tipo II (34.4\%) con OR = 1.19; osteoporosis (27.8\%) con OR $=0.95$; hipertensión arterial (24.5\%) con OR $=0.58$; hipercolesterolemia $(16.6 \%)$ con $\mathrm{OR}=0.28$; enfermedad renal crónica (13.3\%) con OR 0.20, y con otras diez entidades clínicas en menor frecuencia.

\section{DISCUSIÓN}

La vitamina $\mathrm{D}$ es reconocida como una prohormona con efectos pleiotrópicos. Se considera que su deficiencia tiene una prevalencia alta; relacionándose no sólo con raquitismo, osteomalacia y osteoporosis, sino con un mayor riesgo de diabetes, enfermedades cardiovasculares, oncológicas, infecciosas y autoinmunes. ${ }^{9}$ La 25 -hidroxivitamina D3 es la principal forma circulante de la vitamina $\mathrm{D}$, siendo su medición el mejor indicador de los niveles de esta vitamina. ${ }^{10}$ Ésta es transportada por una proteína de unión hacia el riñón para activarse en el túbulo proximal, donde se hidroliza por la enzima mitocondrial $1 \alpha$-hidroxilasa en el carbono 1 del anillo A, para convertirse en 1-25 dihidroxivitamina D3, siendo ésta la responsable de la mayoría de sus efectos biológicos. ${ }^{11}$

Actualmente, se conoce que existen receptores de vitamina $D$ en al menos 30 sitios extrarrenales, a su vez, la enzima $1 \alpha$-hidroxilasa también es expresada en sitios extrarrenales como la placenta, monocitos, macrófagos, la próstata, las mamas, el colon, el corazón, el cerebro, los queratinocitos, las células $\beta$ del páncreas y las glándulas paratiroides, lo que explica que la vitamina $\mathrm{D}$ tenga efectos pleitrópicos y su deficiencia se pueda ver asociada con múltiples enfermedades, por lo que, al día de hoy, se investiga las variadas y múltiples acciones de la vitamina $D$ más allá de sus funciones clásicas sobre el metabolismo del calcio y la salud musculoesquelética. Esto es debido a que la mayoría de los tejidos corporales tienen receptores para la forma activa de 1,25 dihidroxivitamina D2, conocidos como receptores de la vitamina $\mathrm{D}$ (VDR), siendo su gen localizado en el cromosoma 12 (12q13.11). De acuerdo
Tabla 2: Relación de 57 pacientes con 25-OH vitamina D por debajo de $30 \mathrm{ng} / \mathrm{mL}$, y su relación con diagnósticos de solicitud de examen de laboratorio.

\begin{tabular}{|c|c|c|c|c|}
\hline & Diagnóstico & $\mathrm{n}$ & $\%$ & OR \\
\hline 1 & Diabetes Mellitus tipo 2 & 31 & 34.4 & 1.19 \\
\hline 2 & Osteoporosis & 25 & 27.8 & 0.95 \\
\hline 3 & Hipertensión arterial & 22 & 24.5 & 0.58 \\
\hline 4 & $\begin{array}{l}\text { Hipercolesterolemia } \\
\text { tratada con estatinas }\end{array}$ & 15 & 16.6 & 0.28 \\
\hline 5 & $\begin{array}{l}\text { Enfermedad renal } \\
\text { crónica }\end{array}$ & 12 & 13.3 & 0.20 \\
\hline 6 & $\begin{array}{l}\text { Hipotiroidismo en } \\
\text { tratamiento }\end{array}$ & 7 & 7.8 & NC \\
\hline 7 & $\begin{array}{l}\text { Síndrome de ovario } \\
\text { poliquísticos }\end{array}$ & 5 & 5.5 & NC \\
\hline 8 & Anorexia nervosa & 4 & 4.4 & NC \\
\hline 9 & Vigorexia & 3 & 3.3 & $\mathrm{NC}$ \\
\hline 10 & Cáncer de mama & 3 & 3.3 & $\mathrm{NC}$ \\
\hline 11 & Alteración cognitiva & 2 & 2.2 & NC \\
\hline 12 & $\begin{array}{l}\text { Enfermedades de } \\
\text { Graves }\end{array}$ & 2 & 2.2 & NC \\
\hline 13 & $\begin{array}{l}\text { Síndrome de fatiga } \\
\text { crónica }\end{array}$ & 2 & 2.2 & $\mathrm{NC}$ \\
\hline 14 & Enfermedad de Crohn & 1 & 1.1 & NC \\
\hline 15 & Síndrome de DiGeorge & 1 & 1.1 & $\mathrm{NC}$ \\
\hline
\end{tabular}

con el estudio de Bray y su equipo, ${ }^{12}$ la deficiencia de ésta suele presentar polimorfismo; además, la mayoría de estos tejidos también contienen la enzima CYP27B1, responsable de la conversión de la principal forma circulante de la vitamina $D, 25$-hidroxivitamina $D$ en su metabolito activo 1,25 dihidroxivitamina D. La regulación de esta conversión a nivel tisular difiere de la activación convencional que se produce en el riñón en que es más dependiente del sustrato y, por lo tanto, más susceptible a la deficiencia de vitamina D. ${ }^{13}$

En este estudio llama la atención que en $63 \%$ de los casos los niveles séricos de $25-\mathrm{OH}$ vitamina $\mathrm{D}$ se encontraran por debajo de los valores aceptados como normales, especialmente en una población socioeconómicamente alta con acceso a una alimentación, a una educación y atención médica mejores que otras poblaciones; otro de los aspectos es que en todos los casos con vitamina $D$ por debajo del rango normal, se encontró una asociación con alguna entidad clínica, desconociendo si ésta es de manera fortuita, casual o causal; sin embargo, la literatura consultada ha brindado explicación científica en la mayoría de los casos de estas asociaciones. 
Como ejemplos de lo anterior, y sin tomar en cuenta los efectos ampliamente conocidos e investigados sobre el hueso, en varios estudios en animales, desde 1980 se han identificado asociaciones entre los niveles bajos de vitamina D y la diabetes mellitus (DM). Esta asociación está relacionada con la disminución de la secreción de insulina, idea reforzada por la presencia del receptor de vitamina $\mathrm{D}$ y la presencia de proteína de unión a la vitamina $\mathrm{D}$ en tejido pancreático, ${ }^{14,15}$ especialmente en las células $\beta$, así como en varias células del sistema inmune. Se ha comprobado que la vitamina $\mathrm{D}$ modula la expresión de calbindina, la cual participa en el control de flujo del calcio en las células $\beta$, influyendo en la liberación de insulina; además, genera incremento en su síntesis y mejora la sensibilidad de la insulina en los tejidos blancos, reconociendo a la hipovitaminosis $\mathrm{D}$ como uno de los factores de riesgo para diabetes mellitus. ${ }^{16} \mathrm{Al}$ respecto, en el análisis sistemático y metaanálisis se concluye que la vitamina $\mathrm{D}$ en lactantes reduce el riesgo de diabetes mellitus tipo $1 .{ }^{17}$

En diabetes mellitus tipo 2, se ha reportado que los niveles normales de vitamina $D$ mejoran la resistencia a la insulina en prediabetes, ${ }^{18}$ como ejemplo, en el estudio del equipo de Pittas, ${ }^{19}$ en 83,779 enfermeras en seguimiento durante 20 años sin antecedente de diabetes, se encontró que la ingesta diaria de vitamina $\mathrm{D}$ disminuyó el riesgo de diabetes mellitus tipo 2; por otra parte, se reportó que el síndrome metabólico es más frecuente en pacientes con valores bajos de vitamina $\mathrm{D} ;{ }^{20}$ además, múltiples estudios muestran una asociación entre bajas concentraciones de vitamina $\mathrm{D}$ con la intolerancia a la glucosa y la diabetes mellitus tipo $2,^{21-23}$ lo cual está relacionado directamente con acciones de la vitamina $\mathrm{D}$ como la expresión de $1 \alpha$-hidroxilasa en las células $\beta$ pancreáticas y la activación transcripcional del gen de la insulina, que tienen efectos directos e indirectos sobre su secreción, así como la actuación de la insulina sobre los tejidos blancos; esto ha sido corroborado por el trabajo de Mirhosseini y colaboradores. ${ }^{24}$ En el presente reporte, se encontró una asociación entre bajos niveles de vitamina $\mathrm{D}$ y diabetes mellitus en $34.4 \%$ de los casos con OR de 1.19, siendo la única entidad nosológica en donde se demuestra la asociación con niveles bajos $25-\mathrm{OH}$ vitamina $\mathrm{D}$.

En relación con la presencia de enfermedades cardiovasculares, algunos estudios muestran que existe una relación de los bajos niveles circulantes de vitamina $\mathrm{D}$ con el riesgo de hipertensión arterial y enfermedades cardiovasculares; en el presente estudio, la asociación de niveles bajos de vitamina D e hipertensión arterial se encontró en $24.5 \%$ de los casos, con OR $=0.58 .^{25,26} \mathrm{Al}$ respecto, se ha reconocido que la vitamina $\mathrm{D}$ reduce la inflamación relacionada con aterosclerosis, pues se relaciona directamente con el control de varias metaloproteinasas involucradas con la calcificación vascular y los niveles normales de vitamina D, lo que mejora la función de la endotelina, además de que atenúan la hipertrofia de los cardiomiocitos; por otra parte, se ha descrito que la vitamina $\mathrm{D}$ reduce la expresión del gen de renina, disminuyendo, a su vez, la activación de angiotensina I, y modulando en parte al sistema reninaangiotensina-aldosterona. ${ }^{27-29} \mathrm{Al}$ disminuir las concentraciones de vitamina $\mathrm{D}$, la expresión del gen de renina puede verse incrementado y tener su repercusión clínica manifestada como hipertensión arterial.

En pacientes con hipercolesterolemia tratados con estatinas, de acuerdo con el estudio de Bischoff y su equipo, ${ }^{30}$ en análisis longitudinales de pacientes de 60 años o más, manejados con estatinas durante 12 meses, se observó que la concentración de $25-\mathrm{OH}$ vitamina D es significativamente menor en los usuarios de estatinas $(13.1 \mathrm{ng} / \mathrm{mL})$ que en los que no la utilizaron (15.9 ng/mL; $p=0.009)$, ya que varios de ellos utilizaron al sistema de citocromo P450, por lo que pudiera presentarse competencia.

Según la revisión de Pereda y Nishisshinya, ${ }^{31}$ los reportes sugieren que el déficit de vitamina $\mathrm{D}$ incrementa el riesgo de padecer dolor musculoesquelético asociado con el uso de estatinas. Por el contrario, el reporte de Mansur, ${ }^{32}$ menciona que puede elevarse la concentración de vitamina D con el uso de estatinas, por lo que existe controversia al respecto (en el presente trabajo se encontró en $16.6 \%$ de los casos con niveles bajos de vitamina $\mathrm{D}$ asociados con el uso de estatinas, pero el OR correspondió a 0.28).

Respecto a la asociación de vitamina D y cáncer, los estudios epidemiológicos indicaron que los individuos que viven en latitudes más altas tienen una mayor probabilidad de presentar cáncer de colon, próstata, mama, pulmón y esófago. ${ }^{33}$ Garlan y colaboradores ${ }^{34}$ reportaron que valores de $52 \mathrm{ng} / \mathrm{mL}$ de vitamina $D$ reducen la incidencia de cáncer de mama en comparación a niveles menores de $13 \mathrm{ng} / \mathrm{mL}$, al igual que de cáncer de colon, mama y ovario; por otra parte, la 1,25-dihidroxivitamina D3 ejerce efectos de antiproliferación, prodiferenciación, proapoptosis e inhibición de invasión y metástasis, además de inhibir la angiogénesis, haciendo que las células malignas permanezcan en las fases GO/G1 del ciclo celular. ${ }^{35}$ En nuestro estudio, se encontró una asociación de bajos niveles de vitamina $\mathrm{D}$ en $3.3 \%$ de los casos, correspondiendo a cáncer de mama.

Con respecto a la vitamina $\mathrm{D}$ y su asociación con enfermedades de la glándula tiroides, específicamente con hipotiroidismo, ésta fue frecuente. ${ }^{36} \mathrm{El}$ reporte de Torresan y su grupo de investigación ${ }^{37}$ indica que, en 80 mujeres adultas, mayores de 40 años, hipotiroideas controladas con levotiroxina, en la mayoría de la muestra se presentó disminución de valores plasmáticos de 25(OH)D3, observando disminución de los niveles séricos de vitamina $\mathrm{D}$ a medida que aumentaron el peso corporal y la edad. En el 
presente reporte, las concentraciones séricas de vitamina $\mathrm{D}$ se asociaron con $7.8 \%$ de los casos.

Por otra parte, Kitivi y su grupo ${ }^{38}$ documentaron niveles significativamente más bajos de vitamina $\mathrm{D}$ en pacientes con enfermedades tiroides autoinmunes, encontrando que la deficiencia de vitamina $\mathrm{D}$ se relaciona con la presencia de anticuerpos antitiroideos y funciones anormales de la glándula, lo que indica la participación de la vitamina $\mathrm{D}$ en la patogenia en estas entidades, situación apoyada por el reporte de Tetsuyuki y colaboradores. ${ }^{39} \mathrm{Al}$ compararlo con nuestra investigación, se encontró un $2.2 \%$ de los casos con enfermedad de graves asociados con bajos niveles de VD.

El papel de la vitamina D como un modulador inmune se ha enfatizado en los últimos años, y se han observado niveles bajos de vitamina $D$ en varias enfermedades autoinmunes, como esclerosis múltiple, artritis reumatoide y lupus eritematoso sistémico, por lo que, de acuerdo con el trabajo de Blaney y otros, ${ }^{40}$ se ha propuesto a la concentración sérica de vitamina D como un marcador de enfermedades autoinmunes.

La asociación entre niveles bajos de vitamina D y síndrome de ovarios poliquísticos es frecuente, pues, de acuerdo al estudio de Mejía y colaboradores, estos pacientes habitualmente presentan niveles elevados de colesterol, triglicéridos y lipoproteínas de baja densidad; ${ }^{41}$ la suplementación con vitamina $\mathrm{D}$ en estos casos, produce disminución significativa de los tres parámetros mencionados, además de facilitar la acción de la insulina en los órganos blanco. En este trabajo se encontraron cinco casos (5.5\%) con síndrome de ovarios poliquísticos asociados con niveles bajos de vitamina $\mathrm{D}$.

En relación con la asociación de vitamina $\mathrm{D}$ y alteraciones cognitivas, apoya el reporte de Goodwill y Szoeke ${ }^{42}$ sobre una revisión sistemática y metaanálisis del efecto de bajas concentraciones de vitamina D y cognición, que abarcó 26 estudios observacionales y tres estudios de intervención, en donde se reportó que la disminución en los niveles de vitamina $\mathrm{D}$ se asocia con peores estados declinación cognitiva. Con todo, la suplementación de vitamina $\mathrm{D}$ no mostró beneficios estadísticamente significativos en los pacientes con demencia establecida. En el presente reporte, se encontró una asociación de baja de vitamina D con alteraciones cognitivas en dos casos (2.2\%).

En este trabajo, respecto a los niveles bajos de vitamina D y situaciones observadas, como son anorexia nervosa (cuatro casos), vigorexia (tres casos) y enfermedad de Crohn (un caso), éstos pudieran ser explicados por alteraciones en la alimentación, por la no producción de vitamina $\mathrm{D}$ a nivel de la piel, la no absorción de vitamina $\mathrm{D}$ a nivel intestinal y por la disminución del peso corporal con proporción de grasa menor al $85 \%$ del peso corporal (que especialmente en la mujer lleva a menorrea secundaria por baja producción de estrógenos); al respecto, en el reporte de Hamilton, ${ }^{43}$ en 99 adolescentes postmenarcas, con valores de vitamina $D$ bajos inicialmente, la suplementación de vitamina $\mathrm{D}$ alcanzó niveles adecuados mejoraron el salto de altura, la velocidad del salto y la fuerza muscular.

Por último, los bajos niveles de vitamina $\mathrm{D}$ se han considerado en diversos estudios como un posible biomarcador pronóstico en la práctica clínica. En el caso de los adultos mayores hospitalizados en unidades de cuidados geriátricos, las concentraciones séricas más bajas de vitamina $\mathrm{D}$ al momento del ingreso están directamente asociadas con una mayor severidad de las enfermedades crónicas, un mayor riesgo de descompensación aguda y un incremento de la mortalidad hospitalaria. ${ }^{44,45}$

\section{CONCLUSIONES}

En el presente trabajo se puede concluir los siguientes tres aspectos:

1. El número de solicitudes de cuantificación de vitamina $\mathrm{D}$ es muy bajo en la población que es atendida en la institución, quizá porque resulta más económica la prescripción de vitamina $\mathrm{D}$ que su cuantificación por el laboratorio.

2. Los valores bajos de vitamina D se observan en dos tercios de la muestra estudiada, a pesar de que los pacientes corresponden a un estrato socioeconómico alto, en el que, se supone, se tiene acceso a una buena alimentación, educación y control médico.

3. Los valores bajos de vitamina $D$ en este estudio se encontraron asociados con diferentes entidades clínicas, pero solamente su asociación con diabetes mellitus tipo II resultó significativa (con OR de 1.19).

\section{REFERENCIAS}

1. Christakos S1, Ajibade DV, Dhawan P, Fechner AJ, Mady LJ. Vitamin D: metabolism. Endocrinol Metab Clin North Am. 2010; 39: 243-253.

2. Dusso AS, Brown AJ, Slatopolky E. Vitamin D. Am J Physiol Renal Physiol. 20005; 289: F8-28.

3. Lips P, Hosking D, Lippuner K, Norquist JM, Wehren L, Maalouf G, Ragi-Eis $\mathrm{S}$ et al. The prevalence of vitamin $\mathrm{D}$ inadequacy amongst women with osteoporosis: an international epidemiological investigation. J Intern Med. 2006; 260: 245-254.

4. Reichrath J, Lehamann B, Carlberg C, Varani J Varani J, Zouboulis CC. Vitamins as hormones. Horm Metab Res. 2007; 39: 71-84.

5. Forrest KY, Sthuhldreher WL. Ptrevalence and correlates of vitamin D deficiency in US adults. Nutr Res. 2011; 31: 48-54.

6. Kumar J, Muntner P, Kaskel FJ, Hailpern SM, Melamed ML. Prevalence and association of 25-hydroxyvitamin D deficiency in US children. NHANES 2001-20004. Pediatrics. 2009; 124: e362-370.

7. Adams JS, Hewison M Update in vitamin D. J Clin Endocrinol Metab. 2010: 95: 471-478. 
8. Ochoa-Correa EC, García-Hernández PA, Villarreal-Pérez JZ, TreviñoGarza C, Rodríguez-Balderrama I, Martínez-de Villarreal LE et al. Deficiencia de vitamina D en madres y neonatos mexicanos. Gaceta Médica de México. 2017; 153: 559-565.

9. Holick FM, Chen CT. Vitamin D deficiency: a worldwide problem with health consequences. Amer J clinical Nutr. 2008; 87: 10801086.

10. Adams JS, Hewison M Update in vitamin D. J Clin Endocrinol Metab. 2010: 95: 471-478

11. Kochupillai N. The physiology of vitamin D: current concepts. Indian Med Res. 2008; 127: 256-262.

12. Bray M, Hagberg JM, Perusse L, Rankinen T, Roth S, Wofarth B, Bouchard C. The human gene map for performance and healthrelated fitness phenotypes: the 2006-2007 update. Med Sci Sports Exerc. 2009; 41: 34-72.

13. Plum L, DeLuca $H$. The functional metabolism and molecular biology of vitamin D. Clinical Reviews in bone and mineral metabolism. 2009; 7: 20-41.

14. Bloomgarden ZT. American Diabetes Association's 57th annual advanced postgraduate course: disbetes risk; vitamin D, polycystic ovary syndrome, and obstructive sleep apnea. Diabtes Care. 2011; 34: e1-6.

15. Mohr SB, Garland CF, Gorham ED, Garland FC. The association between ultraviolet $\mathrm{B}$ irradiance, vitamin $\mathrm{D}$ status and incidence rates of type 1 diabetes in 51 regions worldwide. Diabtelogia. 2008; 51: 1391-1398.

16. Rocha ML, Baldan da Silva D, Souza AL, Chaim AE, Chaim AE, Pavin EJ, Monte Alegre S. Body composition and metabolic profile in adults with vitamin D deficiency. Rev Nut. 2017; 30: 419-430

17. Zipitis CS, Akobeng AK. Vitamin D supplementation in early childhood and risk of type 1 diabetes: a systematic review and metaanalysis. Arch Dis Child. 2008; 93: 512-517.

18. Mirhosseini N, Vatanparast H, Mazidi M, Kimball MS. Vitamin D Supplementation, Glycemic Control, and Insulin Resistance in Prediabetics: A Meta-Analysis. J Endo Soc. 2018; 2: 687-709.

19. Pittas AG, Lau J, Hu FB, Dawson-Hughes B. The role of vitamin D and calcium in type 2 diabetes. A systematic review and meta-analysis. J Clin Endocrinol Metab. 2007; 92: 2017-2029.

20. Ford ES, Ajani UA, McGuire LC, Liu S. Concentrations of serum vitamin $\mathrm{D}$ and the metabolic syndrome among EUA adults. Diabetes Care. 2005; 28: 1228-1230.

21. Mathiu C, Gysemans C, Giulietti A, Bouillon. Vitamin D and diabetes. Diabetologia. 2005; 48: 1247-1257.

22. Chiu Es, Chu A, Go VL, Saad MF. Hypovitaminosis D is associated with insulin resistence and beta cell dysfunction. Am J Clin Nutr. 2004; 79: 820-825.

23. Teegarden D, Donkin SS. Vitamin D: emerging new roles in insulin sensitivity. Nutr Res Rev. 2009; 22: 82-92.

24. Mirhosseini N, Vatanparast H, Mazidi M, Kimball MS. Vitamin D supplementation, glycemic control, and insulin resistance in prediabetics: A meta-Analysis. J Endo Soc. 2018; 2: 687-709.

25. Scragg R. "Role of vitam D for cardiovascular health". In Holick MF (ed). Vitamin D physiology, molecular biology, and clinical applications. 2a ed. Boston, Humana Press; 2010: 921-936.

26. Zittermann A, Schleithoff SS Koerfer R. Putting cardiovascular disease and vitamin D insufficiency into perspective. Br J Nutr. 2005; 94: 483-492.
27. Li YC. "Vitamin D, renin, and blood pressure". In: Holick MF, ed. Vitamin D physiology, molecular biology, and clinical applications. 2a. ed. Boston, Humana Press; 2010: 937-953.

28. Xiang W, Kong J, Chen S, Cao LP, Qiao G, Zheng W et al. Cardiac hypertrophy in vitamin $\mathrm{D}$ receptor knockout mice: role of the systemic and cardiac renin-angiotensin system. Am J Physiol Endocrinol Metb. 2005; 288: E125- 132.

29. Kim HW, Park CW, Shin YS, Kim YS, Shin SJ, Kim Y et al. Calcitriol regresses cardiac hypertrophy and QT dispersion in secondary hyperparathyroidism on hemodialysis. Nephron Clin Pract.2006; 102: c21-29.

30. Bischoff-Ferrari HA, Fischer K, Orav EJ, Dawson-Hughes B, Meyer U, Chocano-Bedoya PO, Meyer OW et al. Statin use and 25-hydroxyvitamin d blood level response to vitamin $d$ treatment of older. Adults J Am Geriatr Soc. 2017; 65 (6): 1267-1273.

31. Pereda CA, Nishisshinya BM. ¿Existe relación entre los niveles séricos de vitamina D (25OHD) y el dolor musculoesquelético relacionado con la ingesta de estatinas? Revisión sistemática. Reumat Clin. 2016; 12: 303-364.

32. Mansur JL. Efecto de las estatinas sobre la concentración de vitamina d sérica. Arch Med. 2018; 14: 1-4.

33. Holick MF. Vitamin D exraskeletal health. Endocrinol Metab Clin North Am. 2010; 38: 381-400.

34. Garland CF, Gorham ED, Mohr SB, Grant WB, Giovannucci EL, Lipkin $M$. Vitamin D and prevention of breast cancer: pooled analysis. J Steroid Biochem Mol Biol. 2007; 103: 708-711.

35. Wang S. Epidemiology of Vitamin D in health and disease. Nutr Res Rev. 2009; 22: 188-203.

36. Husein MA, Al-ayed BM, Al-rashidi BM. Vitamin D deficiency and its association with thyroid disease. Int J Health Sci (Qassim). 2001; 7: $267-275$

37. Torresan ME, Biasatti C, Cáceres LM, Minniti VP. Estatus de vitamina $\mathrm{D}$ en mujeres adultas hipotiroideas controladas. Rev Arg Endocrin Metab. 2017; 54: 50-54

38. Kivity S, Agmon-Levin N, Zisappl M, Shapira Y, Nagy EV, Dankó K et al. Vitamin D and autoimmune thyroid diseases. Cell Mol Immunol. 2011; 8: 243-247

39. Yasuda T, Okamoto Y, Hamada N, Miyashita K, Takahara M, Sakamoto F, Miyatsuka T et al. Serum vitamin D levels are decreased and associated with thyroid volume in female patients with newly onset Graves' disease. Endocrine. 2012; 42: 739-741.

40. Blaney PG, Albert JP, Proal A. Vitamin D Metabolites as Clinical Markers in Autoimmune and Chronic Disease. Ann N Y Acad Sci. 2009; 1173: 384-390

41. Mejía J, Reyna VE, Andrade AM, Lozada M. Suplementación de vitamina D y perfil lipídico en mujeres con síndrome de ovarios poliquísticos y deficiencia de vitamina D. Medicina Reproductiva y Embriología Clínica. 2018; 5: 123-131.

42. Goodwill MA, Szoeke C. A Systematic Review and meta-analysis of the effect of low vitamin D on cognition. J Am Geriatr Soc. 2017; 65: 2161-2168.

43. Hamilton B. Vitamin D and Human Skeletal Muscle. Scand J Med Science Sport. 2010; 20: 182-190.

44. Gallego GD, Mejía MS, Martínez SL, Rendón DM. Hipovitaminosis D: una visión desde la clínica y la biología molecular. Med UIS. 2017; 30: 45-56.

45. Nair P, Venkatesh B. Vitamin D Deficiency and supplementation in critical illness: the known knowns and known unknowns. Crit Care. 2018; 22: 276. 